\title{
Diseño De Una Planta De Tratamiento De Aguas Residuales Para Su Reutilización En Riego En Áreas Verdes
}

\author{
Mayra Elizabeth Cáceres Mena \\ Carla Sofía Arguello Guadalupe \\ Marco Vinicio Pino Vallejo \\ Galo Briam Montenegro Córdova \\ Catalina Margarita Verdugo Bernal
}

Docente - Investigador de la Facultad de Recursos Naturales ESCUELA

SUPERIOR POLITÉCNICA DE CHIMBORAZO - ECUADOR.

Jaime Giovanny Béjar Suárez.

Docente - Investigador de la Facultad de Ciencias ESCUELA SUPERIOR POLITÉCNICA DE CHIMBORAZO - ECUADOR.

\section{Andrés Paúl Cáceres Mena}

Estudiante, Facultad de Ingeniería ESCUELA SUPERIOR POLITÉCNICA DE CHIMBORAZO - ECUADOR.

doi: 10.19044/esj.2017.v13n18p94 URL:http://dx.doi.org/10.19044/esj.2017.v13n18p94

\begin{abstract}
Over the last few years uncontrolled wastewater discharges have been generated in bodies of water which pose a high risk to the environment and human health.

The present study is based on a laboratory prototype under controlled conditions with the aim: To return water quality indexes to safe conditions within the "Edison Riera" Campus at National University of Chimborazo for its reuse in the green areas irrigation, through unit processes based on the percentages reduction results of polluting organic matter and applying treatment processes by activated sludge.

During the investigation the following results were obtained: Efficiency and reduction of biological and chemical oxygen demand by $93.75 \%$, and $85.02 \%$, coliforms color and turbidity decrease in $87.51 \%, 93.84 \%$ and $90.29 \%$ simultaneously, the same ones that have allowed designing a waste water treatment plant for irrigations in green areas in real scale.
\end{abstract}

Keywords: Wastewater, activated sludge, biological oxygen demand, chemical oxygen demand, turbidity, coliforms 


\section{Resumen}

Durante los últimos años se han generado descargas incontroladas de aguas residuales en cuerpos de aguas que comprometen un alto riesgo para el ambiente y la salud humana.

El presente estudio se basa en un prototipo de laboratorio bajo condiciones controladas con el objeto de: Reconducir los índices de calidad del agua, a condiciones seguras dentro del campus "Edison Riera" de la Universidad Nacional de Chimborazo para su reutilización en el riego de áreas verdes, a través de procesos unitarios en base al resultado de porcentajes de reducción de materia orgánica contaminante y aplicando procesos de tratamiento por lodos activados.

Durante esta investigación se obtuvieron los siguientes resultados: Eficiencia y reducción de la demanda biológica y química de oxígeno en un $93.75 \%$ y $85.02 \%$, disminución de coliformes, color y turbiedad en un $87.51 \%, 93.84 \%$ y $90.29 \%$ simultáneamente, los mismos que han permitido diseñar una planta de tratamiento de aguas residuales para riegos en áreas verdes a escala real.

Palabras clave: Aguas residuales, lodos activados, demanda biológica de oxígeno, demanda química de oxígeno, turbiedad, coliformes

\section{Introducción}

Aproximadamente el cincuenta por ciento de la población a nivel mundial será afectada por la contaminación del agua, debido a una demanda irracional de los recursos hídricos, una gestión deficiente en el manejo del agua y los escasos sistemas de saneamiento (Organización Mundial de la Salud, 2016: 2)

Los procesos de urbanización, industrialización y la agricultura, utilizan una gran cantidad de agua que reducen significativamente los caudales de ríos y las recargas de acuíferos. La falta de políticas y agendas públicas para el tratamiento de aguas residuales en Sur América generan una gran cantidad de residuos líquidos que son vertidos en cuerpos de aguas receptores y en el suelo, generando además de la degradación del ambiente, un alto costo social y afectaciones en la salud pública (Pochat, 2015: 26).

En América latina tan solo el $20 \%$ de las aguas residuales son tratadas aunque la infraestructura que posee la región puede tratar aproximadamente un 30\%. Las principales ciudades de la región cuentan con programas de inversión para el tratamiento de aguas residuales este es el caso de la Ciudad de México, Caracas, Bogotá, Lima, Buenos Aires y Santiago como iniciativa para el control de la contaminación en cuerpos de aguas superficiales y subterráneas (Ballestero Arroyo, \& Mejía, 2015: 27); 
mientras que en Ecuador el tratamiento de aguas residuales es casi inexistente, apenas un $7 \%$ a nivel nacional.

El agua al atravesar diversos procesos antrópicos cambia su composición física, química o biológica, dando origen a las aguas residuales (Ramalho, 2013: 3).

De acuerdo a su origen las aguas residuales contienen altas cargas de materia orgánica, disuelta o en forma coloidal de naturaleza reductora y elementos inorgánicos de distinta composición que parten desde nutrientes hasta sustancias tóxicas y peligrosas. Por esta razón la reutilización de aguas residuales a través de un tratamiento físico, químico o biológico, para el acondicionamiento y aprovechamiento de los recursos hídricos procura generar alternativas e innovación para abastecer las necesidades de actividades económicas y sociales, suponiendo un gran ahorro por el suministro del recurso hídrico, un sistema ecológicamente sustentable y el cumplimiento de los parámetros de calidad para mantener la salud pública (Asano, 2004: 225).

La presente investigación se realizó en el Campus Edison Riera de la Universidad Nacional de Chimborazo (UNACH), donde se utilizó las aguas residuales del campus para determinar los índices de calidad de la misma, y posteriormente realizar pruebas de campo y de laboratorio para el análisis de parámetros físicos, químicos y biológicos.

Las pruebas de campo permiten generar datos reales de las aguas residuales directamente de la descarga en donde se tomaron los siguientes parámetros; oxígeno disuelto, $p \mathrm{H}$, temperatura y conductividad (Crites \& Tchobanaglous, 2000: 26).

Las pruebas de laboratorio de aguas residuales consisten en analizar los siguientes parámetros; $D Q O$ (Demanda química de oxigeno), $D B O$ (Demanda biológica de oxígeno), $\mathrm{pH}$ (Potencial de hidrógeno), turbiedad, sólidos totales, sólidos suspendidos, coliformes totales y fecales. Esta caracterización de campo y laboratorio determinan la cantidad de carga contaminante presente en el efluente (Metcalf \& Eddy, 1995: 60).

La reducción de contaminantes de las aguas residuales mediante las pruebas de tratabilidad fisicoquímicas con la metodología de prueba de jarras y pruebas de tratabilidad biológicas por lodos activados, seleccionan el tratamiento en base a los porcentajes de reducción de los contaminantes dentro de un sistema controlado basado en un prototipo de dosificación continua (Osorio, Torres \& Sánchez, 2010: 4).

La selección del tratamiento eficiente permite proyectar el diseño de una planta de tratamiento de aguas residuales por lodos activados a escala real para cumplir con las condiciones mínimas de calidad del agua para su reúso en el riego de áreas verdes (Rigola, 1989: 43). 


\section{Metodología}

\section{Reconocimiento del lugar y muestreo de aguas residuales}

Se procedió a identificar el punto de muestreo donde las aguas residuales del campus "Edison Riera" son reconducidas a una tubería principal antes de ser descargadas al alcantarillado público de la ciudad de Riobamba. La red de colectores de la urbe comprende un trazado que permite que el sistema de alcantarillado funcione por gravedad, hasta descargar en el pozo de la red sanitaria más cercana que corresponde al barrio "11 de Noviembre".

Una vez identificada la descarga de aguas residuales que se genera en el campus, se determinó las horas para la medición de caudales, en la mañana, tarde y noche utilizando el método volumétrico con la siguiente expresión:

$$
Q=\frac{V}{t}
$$

donde

$Q=$ caudal

$V=$ volumen del fluido

$t=$ tiempo

Se tomaron muestras simples proporcionales al caudal instantáneo de la descarga, brindando así resultados similares a muestras provenientes de un tanque de mezcla completa para el caudal muestreado durante una jornada de trabajo, con el fin de obtener una mayor precisión en la validación de resultados en el laboratorio

(Rigola, 1989: 20).

\section{Pruebas de campo}

La pruebas de campo demuestran particularmente los resultados de la caracterización de efluentes, el muestreo de aguas residuales que inició con la determinación del caudal generado en el Campus UNACH, para luego realizar el análisis in situ de parámetros físico-químicos como temperatura, $p \mathrm{H}$, conductividad y oxígeno disuelto y finalmente la recolección de muestras simples tomadas en la descarga principal a lo largo del día para formar una muestra compuesta del efluente para su traslado y posterior análisis en el laboratorio. El equipo utilizado para medir los parámetros de campo fue el Equipo Multiparámetros HACH HQ 40d.

\section{Análisis en el laboratorio}

Los análisis de laboratorio físico-químicos y biológicos de las muestras compuestas de aguas residuales recolectadas en el campus "Edison Riera" permitieron caracterizar los siguientes parámetros; $p \mathrm{H}$, temperatura, conductividad, oxígeno disuelto, turbidez, sólidos totales disueltos, sólidos suspendidos, sólidos sedimentables, color, demanda 
química de oxígeno, demanda biológica de oxígeno, coliformes fecales y coliformes totales (Shelef, 2006:14).

\section{Pruebas de tratabilidad}

Con las muestras de aguas residuales se realizaron las pruebas de tratabilidad físico-químicas, "pruebas de jarras" y pruebas biológicas por "lodos activados". En las pruebas de jarras se determinó la dosificación óptima de floculantes y coagulantes para disminuir ciertos parámetros de contaminación y en las pruebas por lodos activados se trabajó con un tiempo de aireación continuo las 24 horas al día, por un mes aproximadamente, en donde se fueron analizando los parámetros de oxígeno disuelto, $p \mathrm{H}$, demanda bilógica de oxígeno, demanda química de oxígeno, turbidez, sólidos suspendidos, coliformes fecales y totales (Osorio Robles, Torres Rojo\& Sánchez, 2010:10).

\section{Tratamiento físico-químico (floculación)}

Fue llevado a cabo en el laboratorio de ciencias químicas de la Facultad de Ingeniería, que cuenta con un equipo de jarras provisto de 4 unidades de tratamiento simultáneo. Cada una de estas unidades cuenta con una paleta agitadora que se encarga de generar una mezcla rápida o lenta en las etapas de floculación o coagulación.

Las pruebas de jarras o Jar test, fueron ensayos realizados en laboratorios con aguas residuales; el procedimiento fue: Colocando 4 vasos de $500 \mathrm{ml}$ de agua residual para que sea agitada por las paletas del equipo. La cantidad de coagulante y floculante que se añade a cada uno de los vasos de $500 \mathrm{ml}$ es diferente, y va incrementando hasta encontrar la dosificación necesaria, que logra la coagulación y separación de residuos.

En este ensayo se utilizó sulfato de aluminio como coagulante que permite incrementar la tendencia de las partículas pequeñas y lograr la unión con otras, lo que asegura su sedimentación en poco minutos, con un tiempo de agitación lenta de 1 minuto a $45 \mathrm{rppm}$, y PHP-70 que es un floculante aniónico basado en acrilamida y acrilato de sodio que son tensoactivos de alto peso molecular que se utilizan para separar los sistemas sólidos y líquidos, atrayendo los sólidos presentes en las aguas residuales y precipitándolos, con un tiempo de agitación rápido de 3 a 5 minutos a 110 rppm. Finalmente se deja reposar las aguas tratadas por 5 minutos hasta que se precipiten los sólidos.

\section{Tratamiento biológico (lodos activados)}

El prototipo consistió en un tanque de almacenamiento de agua residual de 70 litros y un tanque de vidrio de $86.00 \mathrm{~cm}$ de largo, $19.00 \mathrm{~cm}$ de ancho y $27.50 \mathrm{~cm}$ de alto, con un volumen útil aproximado de 27.801 , 
dividido en tres compartimientos: Primero un tanque homogeneizador; segundo un tanque de aeración o reactor aeróbico que consta de 4 compresores que proporcionan mezcla completa y aireación al sistema y el tercero un sedimentador. Con volúmenes útiles de 15.78, 7.70 y 4.40 litros, respectivamente. Dichas unidades se comunican a través de orificios de 5 mm de diámetro, ubicados en la parte superior de los tabiques. En la parte media existen salidas con una manguera que permite la extracción de muestras para su análisis. El licor de mezclado y la recirculación de lodo se lo realizan manualmente.

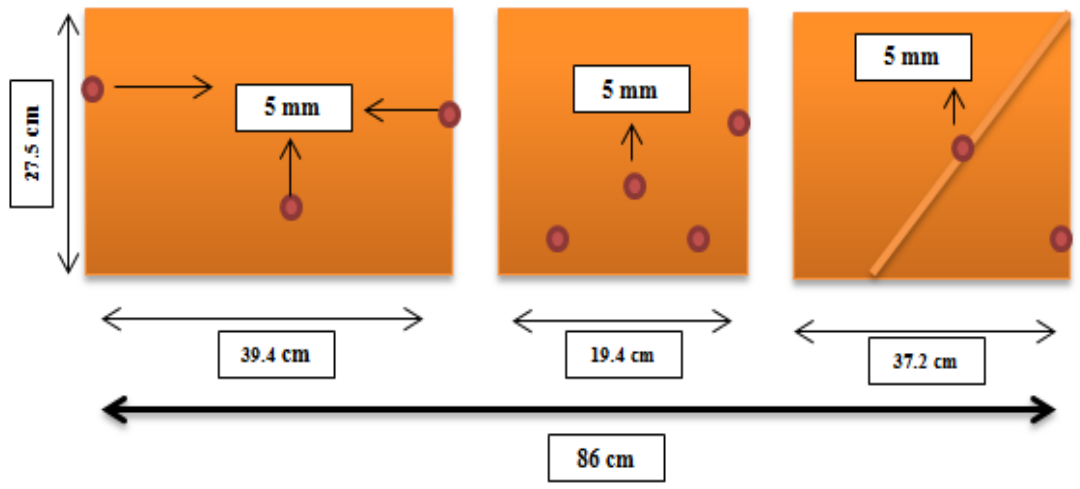

Figura 1: Dimensiones del prototipo Tratamiento biológico

Fuente: Andrés Paúl Cáceres Mena 2015

La operación del sistema se llevó a cabo con la alimentación diaria del agua residual cruda y la recolección del agua tratada después de atravesar el sistema a un tanque de recepción. La determinación del caudal para el sistema en operación se obtiene a partir de la medición del volumen que entra al sistema, de esta manera se obtiene el caudal promedio y se garantiza la representatividad del resultado. El caudal de trabajo fue de 17 $\mathrm{ml} / \mathrm{min}$, con un tiempo de retención de 24 horas por un lapso de 30 días, aproximadamente.

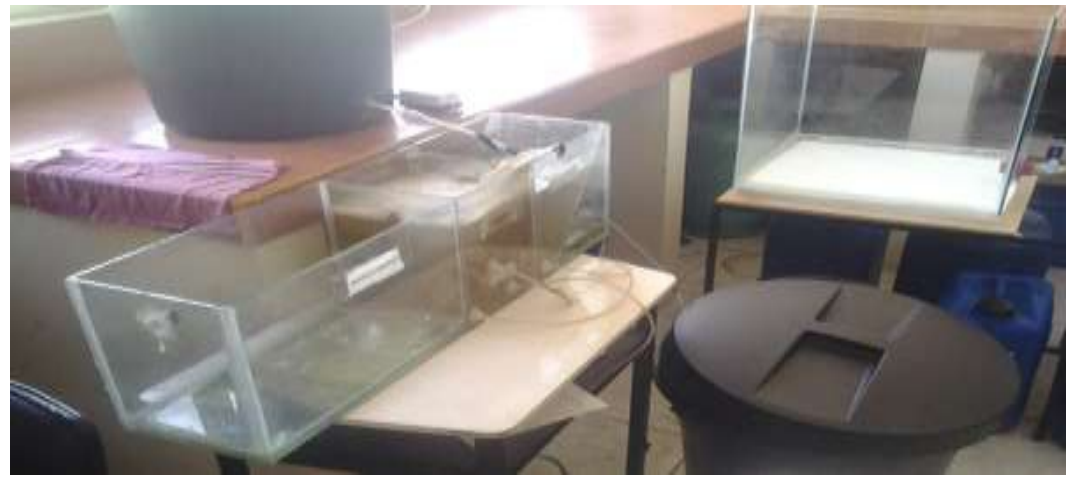

Figura 2: Prototipo para pruebas de tratabilidad biológicas

Fuente: Andrés Paúl Cáceres Mena 2015 


\section{Resultados}

Las características de las aguas residuales del campus UNACH, se expresan en base a promedios, máximos y mínimos, que se han obtenido a partir de diversos análisis realizados con muestras compuestas que provienen de varias muestras simples recolectadas en una jornada diaria de muestreo en la descarga para obtener datos de mayor confiabilidad. A continuación, se presentan los resultados obtenidos de las pruebas de campo, pruebas de laboratorio y pruebas de tratabilidad, de los diferentes parámetros que fueron analizados (Mompin, 1990: 13).

\section{Pruebas de campo}

Los resultados se basan en valores máximos, mínimos, y promedio de los diferentes parámetros in situ que se analizaron durante el muestreo de aguas residuales.

Cuadro 1: Parámetros de medición de campo $p \mathrm{H}$, temperatura (T), oxígeno disuelto (OD) y conductividad

\begin{tabular}{|c|c|c|c|c|}
\hline Valores & $p \mathrm{H}$ & $\mathrm{T}\left[{ }^{\circ} \mathrm{C}\right]$ & OD $[\mathrm{mg} / \mathrm{l}]$ & $\begin{array}{c}\text { Conductividad } \\
{[\mathrm{mS} / \mathrm{cm}]}\end{array}$ \\
\hline Promedio & 8.70 & 17.24 & 2.34 & 2.61 \\
\hline Máximo & 9.00 & 19.70 & 2.62 & 3.18 \\
\hline Mínimo & 8.35 & 13.40 & 2.22 & 2.26 \\
\hline
\end{tabular}

Fuente: Andrés Paúl Cáceres Mena 2015

Con los valores calculados de los parámetros de campo: $p \mathrm{H}$, temperatura, oxígeno disuelto y conductividad, se interpretó los índices de calidad del agua para ser considerada en el uso agrícola en base a las normas ambientales vigentes (McCarty \& Sawyer, 1994: 45).

\section{Resultados de la medición de caudales}

Se expresan los valores promedio, máximos y mínimos obtenidos a través de las mediciones de caudales de la descarga de aguas residuales del Campus Universitario. Estos datos permiten obtener un valor promedio de 55209.06 1/s, para proyectar la propuesta de diseño y dimensionamiento de la planta de tratamiento de aguas residuales. 


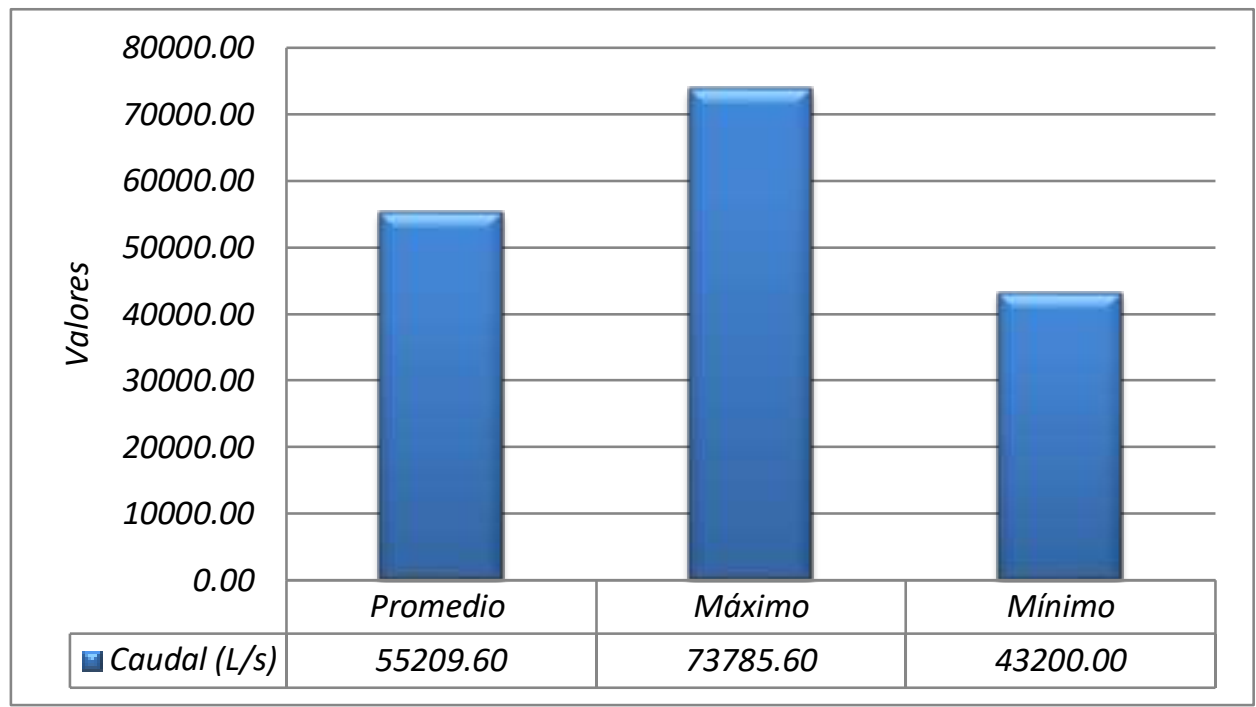

Figura 3: Caudales de las descargas de aguas residuales del campus "Edison Riera" Fuente: Andrés Paúl Cáceres Mena 2015

\section{Pruebas en el laboratorio}

Se procedió a realizar las pruebas de laboratorio de ciertos parámetros que indican el índice de calidad de las aguas residuales y las características de las aguas para definir la tratabilidad de acuerdo a los estándares de calidad para la reutilización en riego.

Cuadro 2: Análisis de Laboratorio

\begin{tabular}{|c|c|c|c|c|}
\hline Parámetros & Promedio & Máximos & Mínimos & Unidades \\
\hline TDS & 1486 & 1888 & 1294 & $\mathrm{mg} / \mathrm{l}$ \\
\hline Turbidez & 69 & 114 & 47 & $\mathrm{FTU}$ \\
\hline$D B O$ & 240 & 291 & 189 & $\mathrm{mg} / \mathrm{l}$ \\
\hline$D Q O$ & 848 & 910 & 680 & $\mathrm{mg} / \mathrm{l}$ \\
\hline $\begin{array}{c}\text { Sólidos } \\
\text { sedimentables }\end{array}$ & 1.8 & 2.5 & 1.5 & $\mathrm{mg} / \mathrm{l}$ \\
\hline Fosfatos & 19.34 & 21.8 & 16.6 & $\mathrm{mg} / \mathrm{l}$ \\
\hline Nitratos & 120 & 125 & 110 & $\mathrm{mg} / \mathrm{l}$ \\
\hline Coliformes fecales & 25720 & 29000 & 22600 & UFC \\
\hline Coliformes totales & 49400 & 52600 & 46700 & UFC \\
\hline
\end{tabular}

Fuente: Andrés Paúl Cáceres Mena 2015

En base al resultado de los análisis se determinaron los parámetros de calidad del agua para definir la tratabilidad de acuerdo a los estándares de calidad que establece la Normativa Ambiental vigente para su reutilizacion en el riego (Ministerio del Ambiente, 2015: 1). 


\section{Pruebas de Tratabilidad}

Las pruebas de tratabilidad se enfocan a la determinación del tratamiento óptimo para las aguas residuales generadas en el campus. Entre éstas tenemos un tratamiento físico-químico o prueba de jarras, y un tratamiento biológico o por lodos activados.

\section{Caracterización de las aguas residuales para las pruebas de tratabilidad}

Se han tomado los parámetros $p \mathrm{H}$, temperatura, turbidez, color $\mathrm{y}$ $D B O$ iniciales de la caracterización del agua cruda para determinar el porcentaje de reducción de los parámetros que actúan como índices de la calidad del agua residual que se genera en el campus.

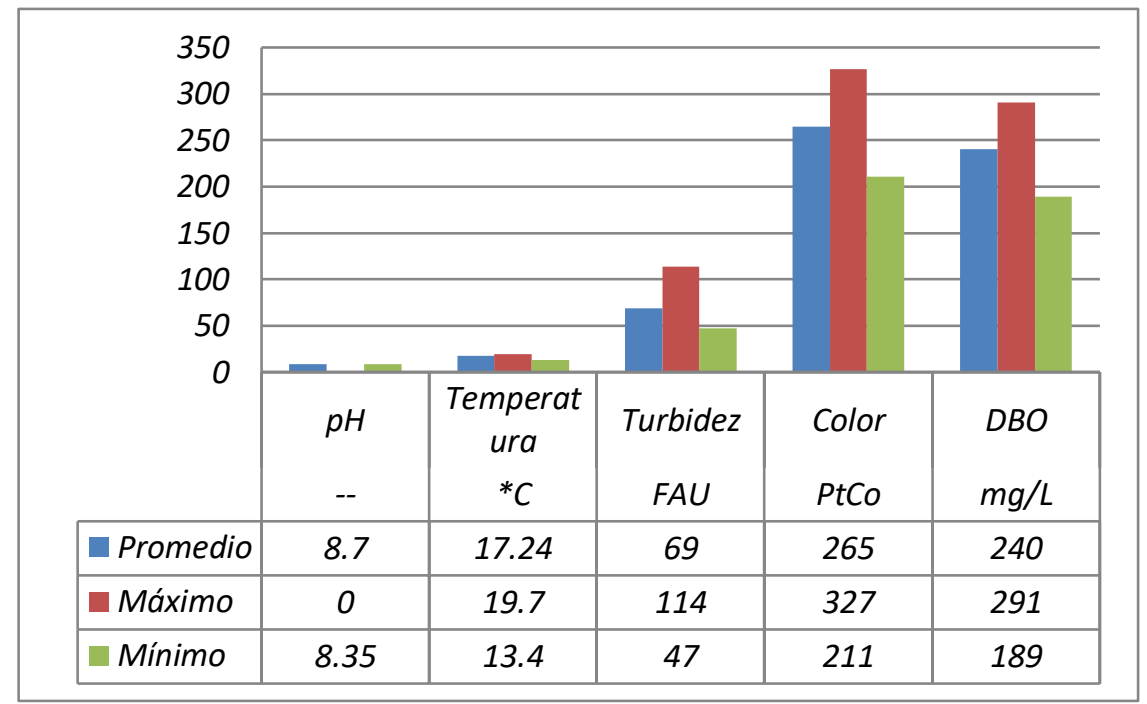

Figura 4: Caracterización de los parámetros del agua residual para el tratamiento físicoquímico

Fuente: Andrés Paúl Cáceres Mena 2015

\section{Prueba de clarificación}

Determinar la dosificación de acuerdo al reactivo utilizado para reducir los parámetros de contaminación establecidos.

Cuadro 3: Dosificación óptima de coagulantes en el tratamiento físico-químico

\begin{tabular}{|c|c|c|c|c|c|}
\hline $\begin{array}{c}\text { Reactivo } \\
\text { Coagulante } \\
\text { Usado }\end{array}$ & $\begin{array}{c}\text { Proporción } \\
(\mathrm{ppm})\end{array}$ & $\begin{array}{c}\text { Floculante } \\
\text { PHP 70 } \\
(\mathrm{ppm})\end{array}$ & $\begin{array}{c}\text { Porcentaje de } \\
\text { Reducción } \\
D Q O\end{array}$ & $\begin{array}{c}\text { Porcentaje } \\
\text { de } \\
\text { Reducción } \\
\text { Color }\end{array}$ & $\begin{array}{c}\text { Porcentaje } \\
\text { de } \\
\text { Reducción } \\
\text { Turbidez }\end{array}$ \\
\hline PAC Chino & 400 & 6 & 77.03 & 91.59 & 91.23 \\
\hline $\begin{array}{c}\text { Sulfato de } \\
\text { Aluminio }\end{array}$ & 200 & 4 & 84.42 & 92.75 & 91.71 \\
\hline
\end{tabular}




\begin{tabular}{|l|l|l|l|l|l|}
\hline $\begin{array}{l}\text { Sulfato } \\
\text { Férrico }\end{array}$ & 300 & 5 & 44.91 & 87.91 & 89.21 \\
\hline
\end{tabular}

Fuente: Andrés Paúl Cáceres Mena 2015

La dosificación adecuada al porcentaje de reducción con coagulante de sulfato de aluminio fue de $200 \mathrm{ppm}$ y $4 \mathrm{ppm}$ de floculante (PHP-70), para lograr la reducción de parámetros físico-químicos. En la demanda química de oxígeno $(D Q O)$ se observó un descenso del $82.42 \%$, el color se redujo en un $92.75 \%$ y finalmente la turbidez con una reducción del $91.71 \%$ en las pruebas de tratabilidad por clarificación.

\section{Pruebas biológicas (lodos activados)}

El tratamiento por lodos activados ha tomado los parámetros que están sobre los límites de la normativa ambiental (MAE, 2015: 25) para realizar el cálculo del rendimiento en porcentaje reducción de contaminantes después del tratamiento a escala.

Cuadro 4: Porcentaje de reducción por aireación prolongada

\begin{tabular}{|c|c|c|c|}
\hline Parámetro & $\begin{array}{c}\text { Concentración de agua } \\
\text { cruda }\end{array}$ & $\begin{array}{c}\text { Concentración de agua } \\
\text { tratada }\end{array}$ & $\begin{array}{c}\text { Porcentaje de } \\
\text { Reducción }\end{array}$ \\
\hline$D B O$ & $240 \mathrm{mg} / \mathrm{l}$ & $15 \mathrm{mg} / \mathrm{l}$ & 93.75 \\
\hline$D Q O$ & $848 \mathrm{mg} / \mathrm{l}$ & $127 \mathrm{mg} / \mathrm{l}$ & 85.02 \\
\hline Color & $1136 \mathrm{ptCo}$ & $70 \mathrm{ptCo}$ & 93.84 \\
\hline Turbidez & $69 \mathrm{NTU}$ & $6.7 \mathrm{NTU}$ & 90.29 \\
\hline Coliformes & $49400 \mathrm{UFC}$ & $6170 \mathrm{UFC}$ & 87.51 \\
\hline
\end{tabular}

Fuente: Andrés Paúl Cáceres Mena 2015

El tratamiento por aireación prolongada o lodos activados reduce el 93.75\% de la demanda biológica de oxígeno $(D B O)$, la demanda química de oxígeno $(D Q O) 85.02 \%$, que son los principales parámetros que indican el estado físico-químico y biológico del agua y del $85 \%$ al $94 \%$ de los demás parámetros que han sido reducidos por los microorganismos degradadores, cumpliendo así con el índice de calidad de aguas para riego de acuerdo a las normativas ambientales vigentes, establecidas en las Tulas.

\section{Selección del tratamiento}

Selección del tratamiento óptimo de acuerdo al porcentaje de reducción de los parámetros físico-químicos y biológicos

Comparando los porcentajes de reducción de los parámetros físicoquímicos y biológicos de las aguas residuales tratadas se selecciona del tratamiento óptimo. 


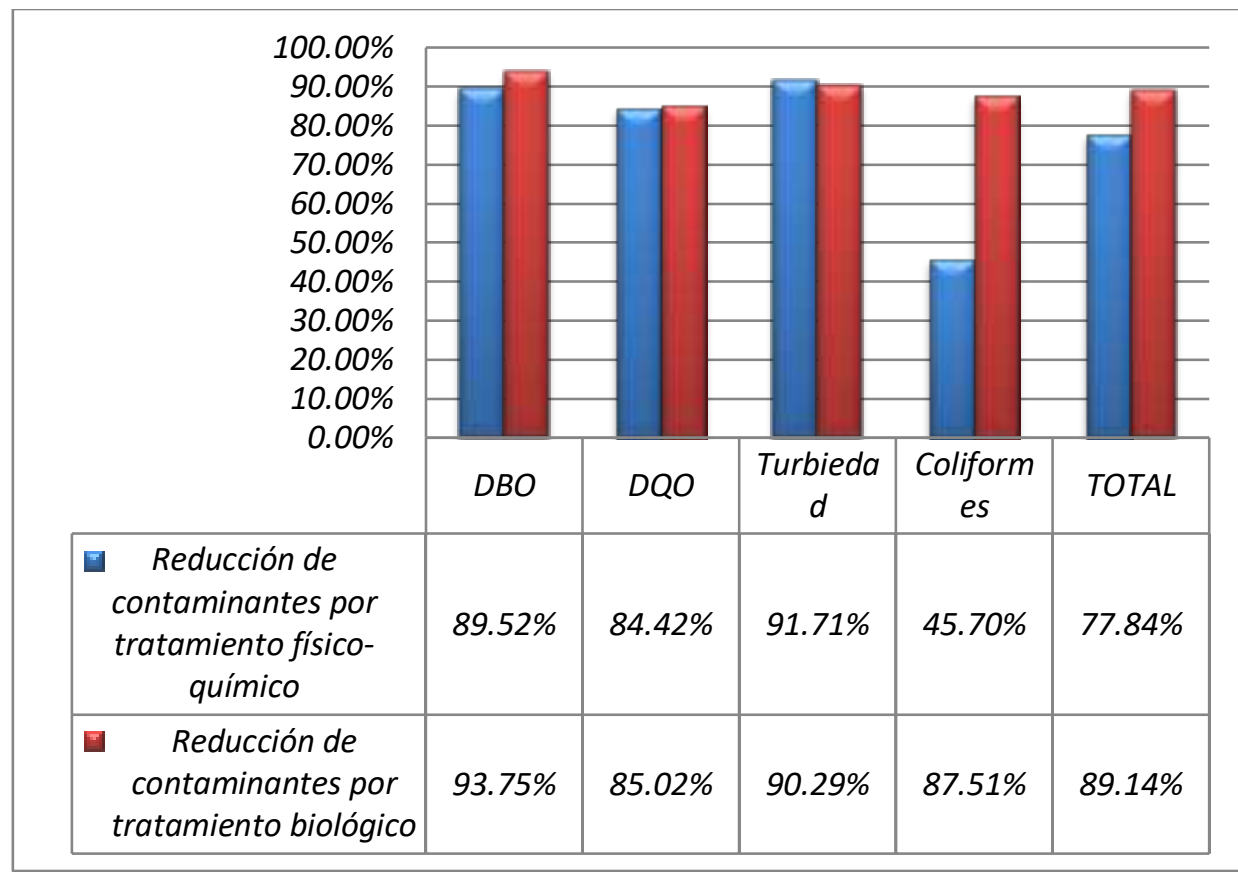

Figura 5: Comparación de los tratamientos de acuerdo al porcentaje de reducción Fuente: Andrés Paúl Cáceres Mena 2015

De acuerdo a la selección del tratamiento y al porcentaje de reducción de los parámetros en el tratamiento físico-químicos cuenta con el $77.84 \%$ de reducción y el tratamiento biológico tiene un $89.14 \%$ de reducción. Por lo tanto, se sugiere el tratamiento biológico por lodos activados, por ser el de mejor resultado.

\section{Relación de los resultados con los límites permisibles establecidos en el marco jurídico ecuatoriano}

En la tabla 5 se establecen los valores comparativos de la concentración inicial del agua cruda y la concentración final de las aguas tratadas mediante un tratamiento biológico a escala obtenido en el laboratorio. A continuación se determina el cumplimiento o no de los límites permisibles establecidos dentro del marco jurídico ecuatoriano (Ministerio del Ambiente, 2015 : 1).

Cuadro 5: Comparación de los parámetros del agua cruda y tratada con la normativa ambiental

\begin{tabular}{|c|c|c|c|c|c|}
\hline Parámetro & Unidad & $\begin{array}{c}\text { Concentración } \\
\text { inicial }\end{array}$ & $\begin{array}{c}\text { Concentración } \\
\text { final }\end{array}$ & $\begin{array}{c}\text { Límite } \\
\text { permisible }\end{array}$ & Cumplimiento \\
\hline$D B O$ & {$[\mathrm{mg} / \mathrm{l}]$} & 240 & 15 & 100 & Cumple \\
\hline$D Q O$ & {$[\mathrm{mg} / \mathrm{l}]$} & 848 & 127 & 250 & Cumple \\
\hline Color & {$[\mathrm{ptCo}]$} & 1136 & 70 & 100 & Cumple \\
\hline Turbidez & {$[\mathrm{NTU}]$} & 69 & 6.7 & $<10$ & Cumple \\
\hline
\end{tabular}

Fuente: Andrés Paúl Cáceres Mena 2015 
Los parámetros de las aguas residuales tratadas cumplen con la normativa ambiental, esto quiere decir que el agua tratada puede ser reutilizada para el riego de áreas verdes.

\section{Diseño de la planta de tratamiento de aguas residuales}

La propuesta planteada consiste en dimensionar los procesos unitarios para el tratamiento de aguas residuales en base al caudal generado en el Campus UNACH y en base a los resultados de la caracterización y las pruebas de tratabilidad realizadas en el laboratorio para calcular y proyectar el diseño a escala real para un tratamiento biológico por lodos activados (Gerard, 2007: 3).

Las fórmulas y ecuaciones para la proyección de los procesos unitarios de la planta de tratamiento fueron tomados del libro de (Romero, 2000: 18).

\section{Diseño de cribas o rejillas}

Cuadro 6: Datos para el dimensionamiento de las cribas o rejillas

\begin{tabular}{|c|c|c|c|}
\hline \multicolumn{3}{|c|}{ Datos iniciales } \\
\hline Parámetro & Simbología & Cantidad & Unidad \\
\hline Caudal & $Q$ & 0.000703 & {$\left[\mathrm{~m}^{3} / \mathrm{s}\right]$} \\
\hline $\begin{array}{c}\text { Velocidad mínima a través de las } \\
\text { barras }\end{array}$ & $V_{b}$ & 0.6 & {$[\mathrm{~m} / \mathrm{s}]$} \\
\hline $\begin{array}{c}\text { Ancho del canal de llegada } \\
\text { Altura de seguridad }\end{array}$ & $B$ & 0.4 & {$[\mathrm{~m}]$} \\
\hline $\begin{array}{c}\text { Ángulo de inclinación de las } \\
\text { barras }\end{array}$ & $H_{s}$ & 0.80 & {$[\mathrm{~m}]$} \\
\hline Separación entre barras & $E$ & 45 & -- \\
\hline Espesor de las barras & $S$ & 0.025 & {$[\mathrm{~m}]$} \\
\hline Coeficiente de pérdidas & $B$ & 0.01 & {$[\mathrm{~m}]$} \\
\hline
\end{tabular}

Fuente: Andrés Paúl Cáceres Mena 2015

Cuadro 7: Resultados para el dimensionamiento de cribas o rejillas

\begin{tabular}{|c|c|c|}
\hline & Resultados & Cantidad \\
\hline Parámetro & $A_{l}={ }^{Q} / V_{b}$ & $0.0012\left[\mathrm{~m}^{2}\right]$ \\
\hline Área libre al paso de agua & $h=\frac{A_{l}}{b}$ & $0.003[\mathrm{~m}]$ \\
\hline Tirante de agua en el canal & $H=h+H_{s}$ & $0.803[\mathrm{~m}]$ \\
\hline Altura total del canal & $L_{b}=\frac{H_{s}}{\sin \propto}$ & $0.94[\mathrm{~m}]$ \\
\hline Longitud de las barras & & \\
\hline
\end{tabular}




\begin{tabular}{|c|c|c|}
\hline Número de barras & $n=\left(\frac{b}{e+S}\right)-1$ & $10--$ \\
\hline Pérdida de carga en las rejillas & $h_{f}=\beta\left(\frac{S}{e}\right)^{4 / 3} * \frac{V_{b}}{2 g} \sin \propto$ & $0.0032[\mathrm{~m}]$ \\
\hline
\end{tabular}

Fuente: Andrés Paúl Cáceres Mena 2015

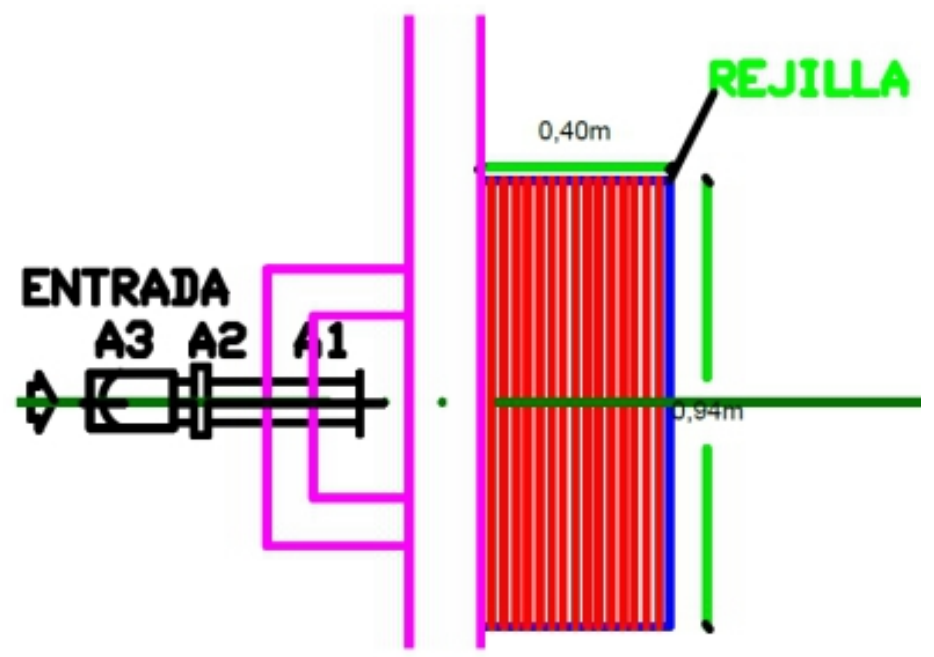

Figura 6: Diseño de las rejillas

Fuente: Andrés Paúl Cáceres Mena 2015

Cuadro 8: Datos para el dimensionamiento del tanque homogenizador

\begin{tabular}{|c|c|c|c|}
\hline \multicolumn{4}{|c|}{ Datos iniciales } \\
\hline Parámetro & Simbología & Cantidad & Unidad \\
\hline Caudal & $Q$ & 55.21 & {$\left[\mathrm{~m}^{3} /\right.$ día] } \\
\hline Tiempo & $\theta$ & 0.5 & [día] \\
\hline
\end{tabular}

Fuente: Andrés Paúl Cáceres Mena 2015

Cuadro 9: Resultados para el dimensionamiento del tanque homogenizador

\begin{tabular}{|c|c|c|}
\hline & Resultados & Cantidad \\
\hline Parámetro & Ecuación & $60.72\left[\mathrm{~m}^{3} / \mathrm{día}\right]$ \\
\hline Caudal & $v=Q * \theta$ & $30.36\left[\mathrm{~m}^{3}\right]$ \\
\hline Volumen & $v=2 h * h * \mathrm{~h} \longrightarrow h=\sqrt[3]{\frac{v}{2}}$ & $2.48[\mathrm{~m}]$ \\
\hline Altura del tanque & $a=2 h$ & $4.96[\mathrm{~m}]$ \\
\hline Largo del tanque & $p=h$ & $2.48[\mathrm{~m}]$ \\
\hline Profundidad & -- & $0.30[\mathrm{~m}]$ \\
\hline Borde libre & 0 & \\
\hline
\end{tabular}

Elaborado por: Andrés Paúl Cáceres Mena 2015 


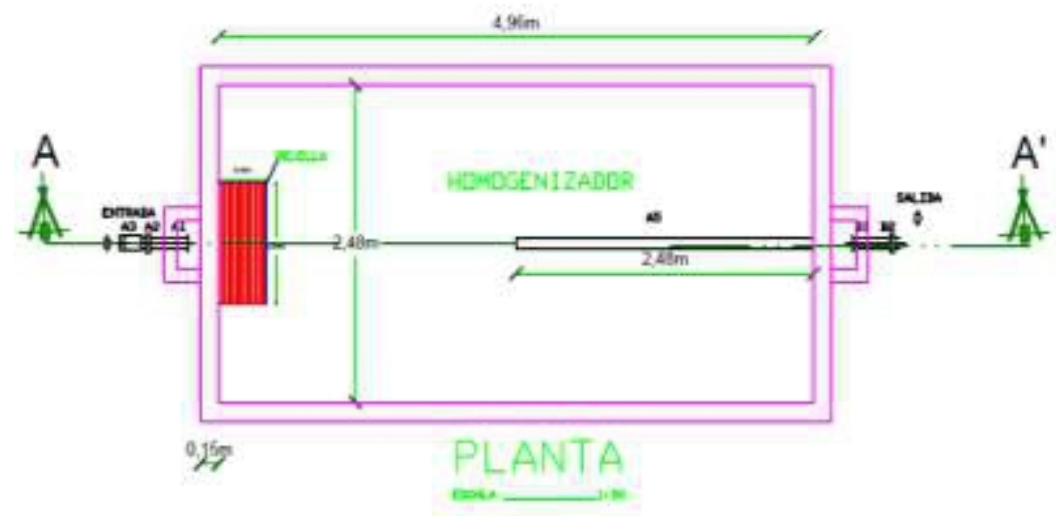

Figura 7: Diseño del tanque homogenizador

Fuente: Andrés Paúl Cáceres Mena 2015

\section{Diseño del sedimentador primario}

Cuadro 10: Datos para el dimensionamiento del sedimentador primario

\begin{tabular}{|c|c|c|c|}
\hline \multicolumn{4}{|c|}{ Datos iniciales } \\
\hline Parámetro & Simbología & Cantidad & Unidad \\
\hline Caudal & $Q$ & 55.21 & {$\left[\mathrm{~m}^{3} / \mathrm{día}\right]$} \\
\hline Tiempo de retención & $\theta$ & 2.00 & {$[$ horas $]$} \\
\hline
\end{tabular}

Fuente: Andrés Paúl Cáceres Mena 2015

Cuadro 11: Resultados para el dimensionamiento del sedimentador primario

\begin{tabular}{|c|c|c|}
\hline \multicolumn{2}{|c|}{ Resultados } \\
\hline Parámetro & Ecuación & Cantidad \\
\hline Volumen & $v=Q * \theta$ & $4.6\left[\mathrm{~m}^{3]}\right.$ \\
\hline Altura del tanque & $v=2 h * h * h \quad \rightarrow h=\sqrt[3]{\frac{v}{2}}$ & $1.32[\mathrm{~m}]$ \\
\hline Largo del tanque & $a=2 h$ & $2.44[\mathrm{~m}]$ \\
\hline Profundidad & $p=h$ & $1.32[\mathrm{~m}]$ \\
\hline Borde libre & -- & $0.30[\mathrm{~m}]$ \\
\hline
\end{tabular}

Fuente: Andrés Paúl Cáceres Mena 2015 


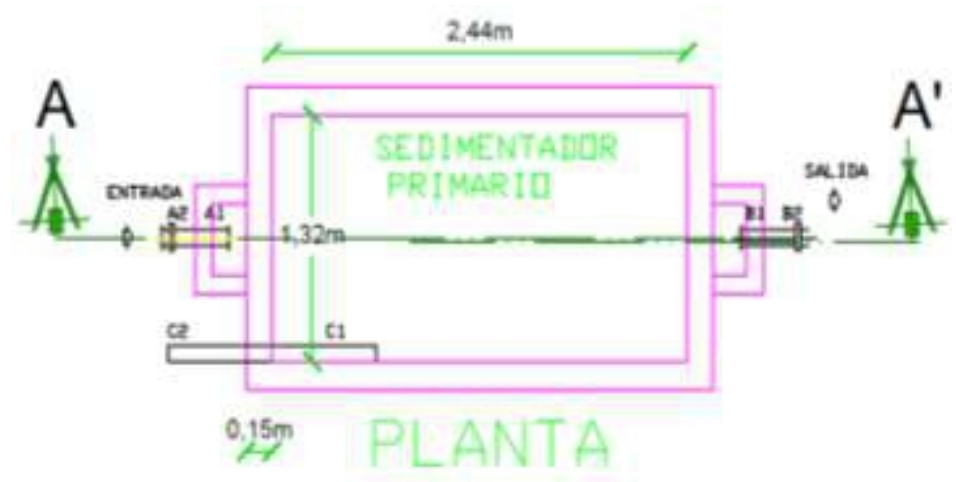

Figura 8: Diseño del sedimentador primario

Fuente: Andrés Paúl Cáceres Mena 2015

\section{Diseño del tanque de aireación}

El tanque de aireación provee de una mezcla de lodos activados recirculados del sedimentador secundario y un flujo de oxígeno provisto de un compresor al agua residual cruda para degradar la materia orgánica debido a la proliferación de bacterias degradadoras aeróbicas en el tanque (Romero, 2000: 15).

Cálculos de los parámetros de las aguas residuales obtenidas en el laboratorio proyectando al caudal real generado en el campus "Edison Riera UNACH":

Cuadro 12: Proyección de los parámetros

\begin{tabular}{|c|c|c|c|}
\hline \multicolumn{4}{|c|}{ Datos iniciales } \\
\hline Parámetro & Simbología & Cantidad & Unidad \\
& & & \\
\hline Caudal & $Q$ & 60.73 & {$[\mathrm{~m} / \mathrm{día}]$} \\
\hline$D B O$ del afluente & $S_{o}$ & 240 & {$[\mathrm{mg} / \mathrm{l}]$} \\
\hline Ssv licor de mezcla & $X$ & 2000 & {$[\mathrm{mg} / \mathrm{l}]$} \\
\hline Tiempo de retención & $\theta$ & 1 & {$[\mathrm{día}]$} \\
\hline$D B O$ del efluente & $S$ & 15 & {$[\mathrm{mg} / \mathrm{l}]$} \\
\hline Sólidos suspendidos del & $S$ & 19 & {$[\mathrm{mg} / \mathrm{l}]$} \\
\hline efluente & $\% S S v$ & 76 & $\%$ \\
\hline Sólidos totales efluente & $S t e$ & 9500 & {$[\mathrm{mg} / \mathrm{l}]$} \\
\hline Constante cinética & $Y$ & 0.91 & -- \\
\hline Constante cinética & $K d$ & 0.01 & - \\
\hline$\%$ O ${ }_{2}$ en el aire & $\% O_{2}$ & 21 & $\%$ \\
\hline Densidad del aire & $d$ & 0.86 & {$\left[\mathrm{~kg} / \mathrm{m}^{3}\right]$} \\
\hline
\end{tabular}

Fuente: Andrés Paúl Cáceres Mena 2015 
Cuadro 13: Resultados obtenidos

\begin{tabular}{|c|c|c|}
\hline \multicolumn{3}{|c|}{ Resultados obtenidos } \\
\hline Parámetro & Ecuación & Cantidad \\
\hline $\begin{array}{l}\text { Tiempo de retención } \\
\text { hidráulica o tiempo de } \\
\text { aireación }\end{array}$ & $\theta=\frac{v}{Q}$ & 1 [día] \\
\hline $\begin{array}{c}\text { Carga orgánica del } \\
\text { proceso }\end{array}$ & $C_{o}=S_{o} * Q$ & $14.37[\mathrm{mg} / \mathrm{l}]$ \\
\hline $\begin{array}{c}\text { Carga orgánica } \\
\text { volumétrica }\end{array}$ & $C_{o v}=\frac{C_{o}}{V}$ & $0.48\left[\frac{\mathrm{kg} * \mathrm{DBO}}{\mathrm{m}^{3} * \text { día }}\right]$ \\
\hline$D B O$ del efluente & $S_{e}=D B O_{v}-0.63$ & $14.575[\mathrm{mg} / \mathrm{l}]$ \\
\hline Biomasa en el reactor & $X V=\left(Y * Q\left(S_{o}-S_{e}\right) /\left(1+K_{d}+\theta_{c}\right)\right.$ & $115.743[\mathrm{~kg} \mathrm{Ssv}]$ \\
\hline Producción de lodo & $P_{x}=\frac{X V}{\theta_{c}}$ & $\begin{array}{l}11.57 \text { [kg } \\
\text { Ssv/día] }\end{array}$ \\
\hline $\begin{array}{c}\begin{array}{c}\text { Sólidos totales de } \\
\text { desecho }\end{array} \\
\end{array}$ & $L_{s}=\frac{P_{x}}{\% S s v}$ & 15.23 [kg/día] \\
\hline Caudal de lodos & $Q_{w}=L_{S} / S_{t e}$ & 1.6 [m³/día] \\
\hline $\begin{array}{c}\text { Sólidos de } \\
\text { recirculación }\end{array}$ & $X r=\% S S V * S_{t e}$ & $7220[\mathrm{mg} / \mathrm{l}]$ \\
\hline $\begin{array}{l}\text { Caudal de } \\
\text { recirculación }\end{array}$ & $Q_{r}=\frac{Q * X}{X_{r}-X}$ & 23.27 [m³/día $]$ \\
\hline Demanda de oxígeno & $D O=1.5 * Q\left(S_{o}-S_{e}\right)-1.42 * X_{r} * Q_{w}$ & $4.13\left[\mathrm{~kg} \mathrm{O}_{2} /\right.$ día $]$ \\
\hline Caudal de aire & $Q_{\text {aire }}=\frac{D O}{\% O * \delta}$ & $22.87\left[\mathrm{~m}^{3} /\right.$ día $]$ \\
\hline $\begin{array}{l}\text { Relación alimento / } \\
\text { Microorganismo }\end{array}$ & $\frac{A}{M}=\frac{Q * S_{o}}{X V}$ & $0.13\left[\mathrm{~d}^{-1}\right]$ \\
\hline $\begin{array}{l}\text { Eficiencia en } \\
\text { reducción } D B O \text { total }\end{array}$ & $E=\frac{S_{o}-S_{e}}{S_{o}}$ & $97.17 \%$ \\
\hline $\begin{array}{l}\text { Eficiencia en } \\
\text { reducción } D B O \\
\text { soluble }\end{array}$ & $E T=\frac{S_{o}-S_{e}}{S_{o}}$ & $93.75 \%$ \\
\hline
\end{tabular}

Fuente: Andrés Paúl Cáceres Mena 2015

Dimensionamiento de la planta de tratamientos

Cuadro 14: Datos para el dimensionamiento del tanque de aireación

\begin{tabular}{|c|c|c|c|}
\hline \multicolumn{4}{|c|}{ Datos iniciales } \\
\hline Parámetro & Simbología & Cantidad & Unidad \\
\hline Caudal ingreso & $Q_{i}$ & 60.73 & {$\left[\mathrm{~m}^{3} / \mathrm{dí}\right]$} \\
\hline
\end{tabular}




\begin{tabular}{|c|c|c|c|}
\hline Caudal de recirculación & $Q_{r}$ & 23.27 & {$\left[\mathrm{~m}^{3} /\right.$ día] } \\
\hline Tiempo & $\theta$ & 1.00 & [día] \\
\hline
\end{tabular}

Fuente: Andrés Paúl Cáceres Mena 2015

Cuadro 15: Resultados para el dimensionamiento del tanque de aireación

\begin{tabular}{|c|c|c|}
\hline \multicolumn{2}{|c|}{ Resultados } \\
\hline Parámetro & Ecuación & Cantidad \\
\hline Caudal total & $Q T=Q_{i}+Q_{r}$ & $84\left[\mathrm{~m}^{3} / \mathrm{día}\right]$ \\
\hline Volumen & $v=Q * \theta$ & $84\left[\mathrm{~m}^{3}\right]$ \\
\hline Altura del tanque & $v=2 h * h * h \quad-\quad \square=\sqrt[3]{\frac{v}{2}}$ & $3.48[\mathrm{~m}]$ \\
\hline Largo del tanque & $a=2 h$ & $6.96[\mathrm{~m}]$ \\
\hline Profundidad & $p=h$ & $3.48[\mathrm{~m}]$ \\
\hline Borde libre & -- & $0.30[\mathrm{~m}]$ \\
\hline
\end{tabular}

Fuente: Andrés Paúl Cáceres Mena 2015

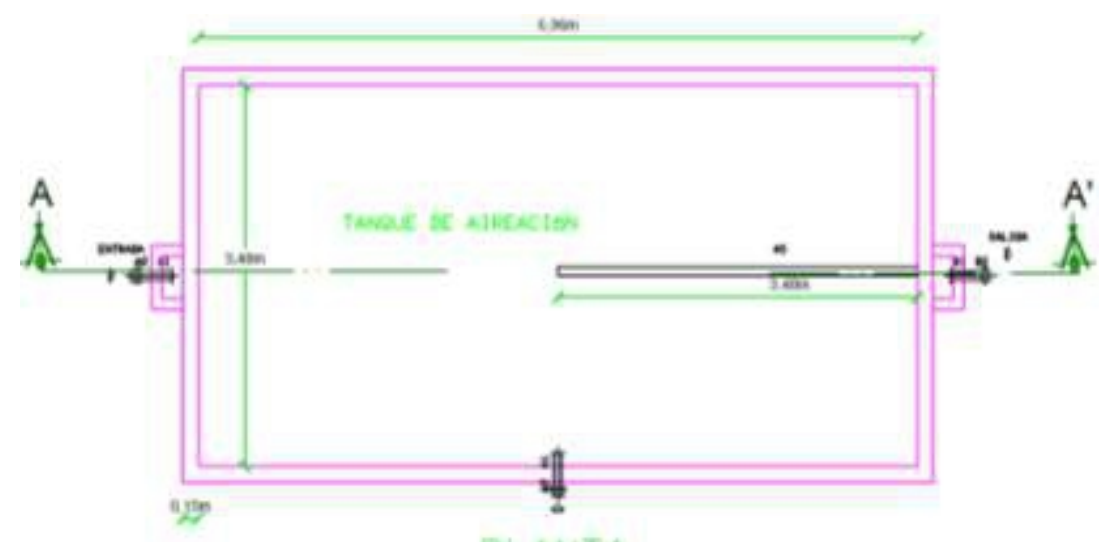

Figura 9: Diseño del tanque de aireación

Fuente: Andrés Paúl Cáceres Mena 2015

\section{Diseño del sedimentador secundario}

Cuadro 16: Datos para el dimensionamiento del sedimentador secundario

\begin{tabular}{|c|c|c|c|}
\hline \multicolumn{4}{|c|}{ Datos iniciales } \\
\hline Parámetro & Simbología & Cantidad & Unidad \\
\hline Caudal & $Q$ & 84 & {$\left[\mathrm{~m}^{3} / \mathrm{día}\right]$} \\
\hline Tiempo de retención & $\theta$ & 2.00 & {$[$ horas] } \\
\hline
\end{tabular}

Fuente: Andrés Paúl Cáceres Mena 2015

Cuadro 17: Resultados para el dimensionamiento del sedimentador secundario

\begin{tabular}{|l|l|l|}
\hline \multicolumn{2}{|l|}{ Resultados } & Cantidad \\
\hline Parámetro & Ecuación & Canto \\
\hline
\end{tabular}




\begin{tabular}{|c|c|c|}
\hline Volumen & $v=Q * \theta$ & $7\left[\mathrm{~m}^{3}\right]$ \\
\hline Altura del tanque & $v=2 h * h * h \quad \longrightarrow \quad h>\sqrt[3]{\frac{v}{2}}$ & $1.52[\mathrm{~m}]$ \\
\hline Largo del tanque & $a=2 h$ & $3.04[\mathrm{~m}]$ \\
\hline Profundidad & $p=h$ & $1.52[\mathrm{~m}]$ \\
\hline Borde libre & -- & $0.30[\mathrm{~m}]$ \\
\hline
\end{tabular}

Fuente: Andrés Paúl Cáceres Mena 2015

Figura 10: Diseño del sedimentador secundario

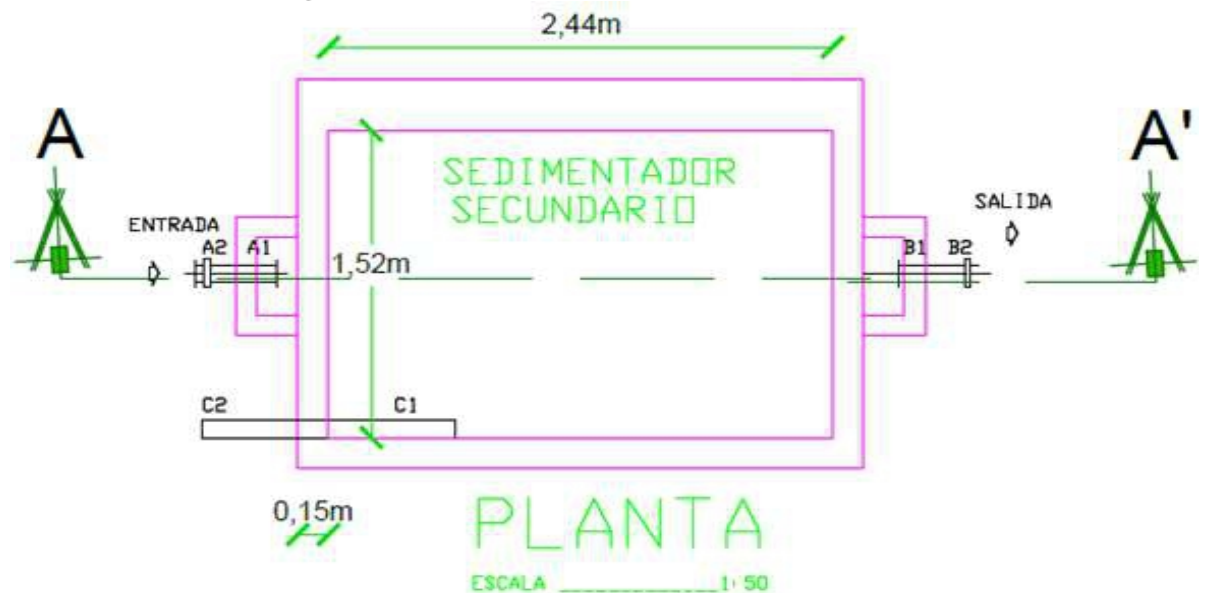

Fuente: Andrés Paúl Cáceres Mena 2015

\section{Diseño del tanque de cloración}

Cuadro 18: Datos para el dimensionamiento del tanque de cloración

\begin{tabular}{|c|c|c|c|}
\hline \multicolumn{3}{|c|}{ Datos iniciales } \\
\hline Parámetro & Simbología & Cantidad & Unidad \\
\hline Caudal & $Q$ & 84 & {$\left[\mathrm{~m}^{3} /\right.$ día $]$} \\
\hline Caudal de recirculación & $Q_{r}$ & 23.27 & {$\left[\mathrm{~m}^{3} /\right.$ día $]$} \\
\hline Caudal de lodos & $Q_{w}$ & 1.6 & {$\left[\mathrm{~m}^{3} /\right.$ día $]$} \\
\hline $\begin{array}{c}\text { Tiempo de retención } \\
\text { Dosis recomendada de } \\
\text { cloro }\end{array}$ & $\theta$ & 1.00 & {$[\mathrm{hora}]$} \\
\hline$C_{2}$ & 8 & {$[\mathrm{mg} / \mathrm{l}]$} \\
\hline
\end{tabular}

Fuente: Andrés Paúl Cáceres Mena 2015

Cuadro 19: Resultados para el dimensionamiento del tanque de cloración

\begin{tabular}{|c|c|c|}
\hline \multicolumn{3}{|c|}{ Resultados } \\
\hline Parámetro & Ecuación & Cantidad \\
\hline Caudal Total & $Q T=Q-Q_{r}-Q_{i w}$ & $59.13\left[\mathrm{~m}^{3} / \mathrm{día}\right]$ \\
\hline Volumen & $v=Q * \theta$ & $2.50\left[\mathrm{~m}^{3}\right]$ \\
\hline Altura del tanque & $v=2 h * h * h \quad \longrightarrow=\sqrt[3]{\frac{v}{2}}$ & $1.08[\mathrm{~m}]$ \\
\hline
\end{tabular}




\begin{tabular}{|c|c|c|}
\hline Largo del tanque & $a=2 h$ & $2.16[\mathrm{~m}]$ \\
\hline Profundidad & $p=h$ & $1.08[\mathrm{~m}]$ \\
\hline Borde libre & -- & $0.30[\mathrm{~m}]$ \\
\hline Consumo diario de cloro & & 0.473 \\
\hline
\end{tabular}

Fuente: Andrés Paúl Cáceres Mena 2015

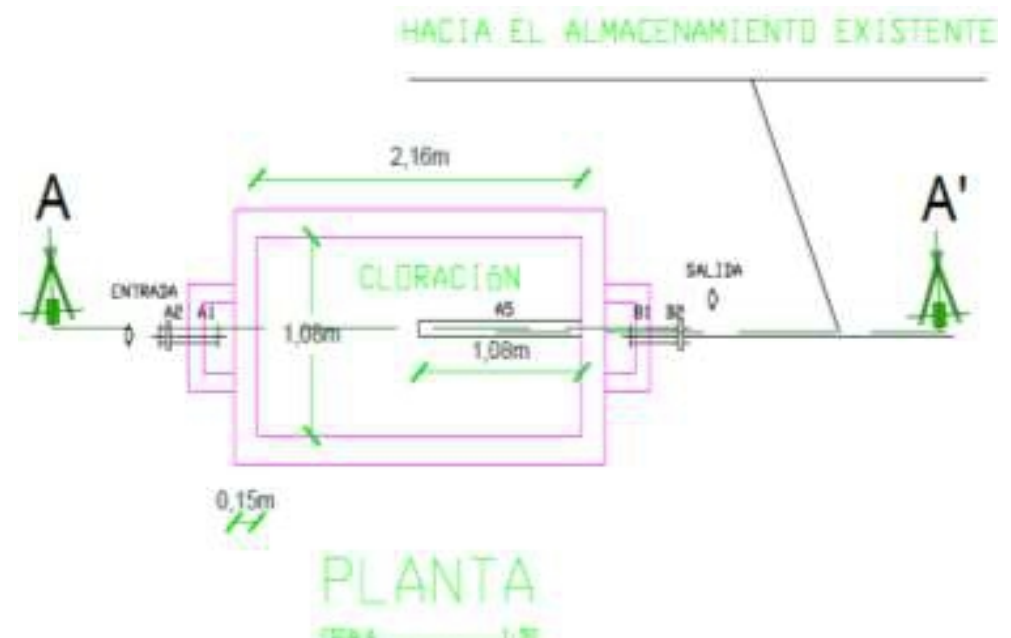

Figura 11: Diseño del tanque de cloración Fuente: Andrés Paúl Cáceres Mena 2015

\section{Conclusiones}

1. El determinar las características físico-químicas y biológicas de las aguas residuales mediante la medición de parámetros in situ y en el laboratorio permite establecer las pruebas de tratabilidad para identificar el sistema de depuración para las aguas residuales generadas en el campus "Edison Riera" y permite conocer el dimensionamiento de las plantas de tratamientos de acuerdo a su caudal promedio de 55 209.6 1/día.

2. El sistema controlado por lodos activados y aireación prolongada ayuda a que los microorganismos aerobios se desarrollen en el sistema y estos se encarguen de descomponer la materia orgánica de las aguas residuales obteniendo porcentajes de reducción de los parámetros de control como la demanda biológica de oxígeno en un 93.75\%, demanda química de oxígeno en un $85.02 \%$, color $93.84 \%$, turbiedad $90.29 \%$, coliformes $87.51 \%$.

3. De las pruebas de laboratorio se determina que el tratamiento de aguas residuales por medio de lodos activados es el tratamiento más eficiente en comparación con los ensayos realizados con clarificación físicoquímica. El cual se ha convertido en un sistema de alto rendimiento que permite que los parámetros sean aptos para la reutilización de las aguas 
tratadas en el riego de áreas verdes de acuerdo a la Ley de Gestión Ambiental (Ministerio de Ambiente, 2015:1).

4. En los ensayos físico-químicos de laboratorio se dosificó floculantes y coagulantes químicos en las aguas residuales para su tratamiento, pero produjo resultados del $77.84 \%$ de reducción, siendo un porcentaje de reducción menor al tratamiento biológico con un porcentaje de reducción del $89.14 \%$.

\section{References:}

1. Asano, L. (2004). Wastewater engineering: treatment and reuse. Londres: McGraw-Hill.

2. Ballestero, M., Arroyo, V., \& Mejía, A. (2015). VII Foro Mundial del Agua. Inseguridad Económica del Agua en Latinoamérica: de la abundancia a la inseguridad (p. 34). Corea: CAF.

3. Crites, R., \& Tchobanoglous, G. (2000). Tratamiento de aguas residuales en pequeñas poblaciones. Bogotá: McGraw-Hill.

4. Gerard, J. (2007). Tratamiento de aguas negras y desechos industriales. México D.F.: UTEHA. p3.

5. McCarty, \& Sawyer. (1994). Regeneración y reutilización de aguas residuales. Berlín: Fundación Agbar.p 45.

6. Metcalf, \& Eddy. (1995). Ingeniería de aguas residuales (Vol. I). Madrid: McGraw-Hill.

7. Ministerio del Ambiente. (2015). Reforma del libro VI del texto unificado de legislación ecuatoriana. Quito, Ecuador: Corporación de Estudios y Publicaciones. p 1.

8. Mompín, G. (1990). Tratamiento de aguas residuales. Madrid: Ediciones Vega. p. 13.

9. Organización Mundial de la Salud. (2016). Planificación de la seguridad del saneamiento. Manual para el uso y la disposición seguros de aguas residuales, aguas grises y excretas. Ginebra: Ediciones de la OMS.

10. Osorio Robles, F., Torres Rojo, J. C., \& Sánchez, M. (2010). Tratamiento de aguas para la eliminación de microorganismos y agentes contaminantes. Madrid: Ediciones Díaz de Santos. p 10.

11. Osorio, F., \& Hontoria, E. (2010). Saneamiento y alcantarillado, vertidos de aguas residuales. Granada: Degremont.

12. Pochat, V. (2015). VII Foro Mundial del Agua. Una agenda del agua para América del Sur: desafíos, visión y estrategias (p. 53). República de Corea: CAF.

13. Ramalho, R. S. (2013). Introduction to the waterwater treatment processes (2nd. ed.). New York: Academic Press. 
14. Rigola, L. (1989). Tratamiento de aguas industriales, aguas de proceso y residuales. Madrid: Boixareu Editores. p 20

15. Romero, J. (2000). Tratamiento de aguas residuales (1ra. ed.). Bogotá: Escuela Colombiana de Ingeniería. p 15-18

16. Shelef, L. (2006). Biotecnología del medio ambiente, principios y aplicaciones. Bogotá: McGraww-Hill. p 14. 